\title{
Magnetic Resonance Diffusion Tensor Tractography in Assessment of Cystic Brain Lesions
}

\author{
Mona Tharwat ${ }^{1}$, Al-Siagy Ali ${ }^{2}$, Ahmed Yousef ${ }^{3}$, Samah Ahmed $^{2}$ \\ ${ }^{1}$ Ministry of Health, Damietta, Egypt \\ ${ }^{2}$ Department of Radiology, Faculty of Medicine, Tanta University, Tanta, Egypt \\ ${ }^{3}$ Department of Neurosurgery, Faculty of Medicine, Tanta University, Tanta, Egypt \\ Email address: \\ thrwtmny3@gmail.com (Mona T.), alsiagy.ali@med.tanta.edu.eg (Al-Siagy A.), samah.radwan@med.tanta.edu.eg (Ahmed Y.), \\ ahmed.soliman@med.tanta.edu.eg (Samah A.) \\ ${ }^{*}$ Corresponding author
}

\section{To cite this article:}

Mona Tharwat, Al-Siagy Ali, Ahmed Yousef, Samah Ahmed. Magnetic Resonance Diffusion Tensor Tractography in Assessment of Cystic Brain Lesions. International Journal of Medical Imaging. Vol. 9, No. 1, 2021, pp. 57-71. doi: 10.11648/j.ijmi.20210901.16

Received: January 17, 2021; Accepted: February 3, 2021; Published: February 10, 2021

\begin{abstract}
Brain cystic lesions is significant neurosurgical dilemma. Differencing cystic brain lesions by CT and conventional MRI may be difficult due to similar findings clinically and similar appearance radiologically, On conventional MRI most of the lesions appear hyper intense on T2-WI and hypo intense on T1-WI. The aim of this study was to assess the role of magnetic resonance diffusion tensor tractography in the assessment and differentiation between cystic brain lesions. The study was a prospective one that was conducted on 30 patients who were suspected to have cystic brain lesions and referred to radio diagnosis department from the neuro-surgery department and outpatient clinics of Tanta university hospitals. In this study mean age of patients was $43.453+12.23$ years, their age ranged from $5-60$ years. DTI guides the surgeons regarding the relation between the intra-axial tumor and local WM tracts in different planes. A variety of aspects of the tumor-tract relationship can, therefore, be revealed. The nature of the tract can be suggested from its position and course, such as the corticospinal tract (CST) and optic radiations, and effect of the tumor to the tract can be appreciated. Furthermore, the location of the tumor can be seen in relation to the tract. Neurosurgery for brain tumors is comparison between maximum surgical resection on one hand and maximum sparing of functions on other hand. Total resection of the tumor reduces risk of relapse and permits following radiotherapy or chemotherapy to be much more effective.
\end{abstract}

Keywords: MRI, Tractography, Cystic Brain Lesions, Diffusion MRI

\section{Introduction}

Brain cystic lesions is obviously neurosurgical dilemma. Differentiation between cystic brain lesions by CT and MRI may be difficult due to similar clinical findings and similar radiologic appearance. Diffusion weighted imaging (DWI) is important diagnostic modality in differentiating brain cystic lesions. As in conventional MRI most of the cystic lesions appear hyper-intense on T2-WI and hypo-intense on T1-WI. Intracranial cystic lesions show rim enhancement on postcontrast T1-WI [1].

On the other hand, identifying the location of the motor pathways has been much more challenging. massive white matter infiltration by primary brain tumors is common [2].

Otherwise, resection of brain tumor carries risk of destroying descending motor pathway. Diffusion tensor (DT)-imaged fiber tracking is a non-invasive magnetic resonance (MR) technique that helps to visualize the white matter fibers such as cortico-spinal (pyramidal) tract, optic radiation and arcuate fasciculus with relationship to cystic lesions and can delineate the subcortical course of the motor pathway [3].

Diffusion tensor imaging (DTI) and white matter tractography (WMT) are advisable techniques to estimate course, extent, and connectivity forms of white matter (WM) structures in human brains. DTI gives details on tissue microstructure and organization better than usual image resolution. 
With diffusion tensor imaging, diffusion anisotropy can detect white matter abnormalities not normally seen on conventional MRI [4].

Pre-operative assessment with (DT)-imaged fiber tracking can be used to demonstrate definite location of targeted white matter fibers \& displacement of the white matter tracts and in assessment of micro-structural patency of white matter seen near to tumors and so it can be useful for neuro-surgical plans to reduce interfering of the white tracts and improve pre-operative risk analysis [5].

Fiber tracks assessed by DTI can be used to identify and navigate trans cranially through motor tracts in deep white matter and define a safety margin around the tract as possible. That grantee the advantage of DT-imaged fiber tracking as an informative pre-surgical planning tool [3].

\section{Patients and Methods}

The present study was a prospective one that was conducted on 30 patients who were suspected to have cystic brain lesions at the period between February 2018 to February 2020.

Patients were referred to radio diagnosis department from the neuro-surgery department and outpatient neurological clinics of Tanta university hospitals with suspected or diagnosed cystic brain lesions and underwent MSCT with contrast to confirm the diagnosis or to answer specific anatomic questions.

Patients were selected according to the following inclusion and exclusion criteria:

\subsection{Inclusion Criteria}

This study included 30 patients known or strongly suspected to have cystic brain lesions with inconclusive CT findings.

\subsection{Exclusion Criteria}

The study ruled out patients with MRI contraindications as: brain clips for aneurysms, implanted neural stimulators, implanted pacemakers for heart or defibrillators, cochlear implants, ocular foreign bodies (e.g. metal shavings) and other implanted devices: (e.g. swan ganz catheter), insulin pumps, metal shrapnel or bullet $\&$ allergic to contrast media

\subsection{Method}

\subsubsection{Every Patient Was Subjected to History Taking and Clinical Examination}

1. At baseline, a medical history and physical examinations were undertaken and following data were obtained: age, sex, smoking status, duration of disease, and presence of hypertension.

2. Detailed history was taken regarding the onset of the disease, the presenting, any previous treatments and their types whether surgical or medical, any previous laboratory investigations, and any previous imaging procedures.
3. The patients were also asked about the history of recurrence and family history. Also detailed history was taken about life style and history of diabetes.

4. General examination \& neurological examination in the form of:

a) Mental state: Speech, dysarthria, aphasia, dysphonia and aphonia.

b) Cranial nerves.

c) Motor system: State, tone, power, fasciculation, involuntary movements, reflexes, superficial reflexes, deep reflexes and coordination.
d) Sensory system.
e) Superficial sensations.
f) Deep sensations.
g) Cortical sensations.
h) Sphincteric disturbances.
i) Gait disturbances.

\subsubsection{Patient Preparation for MRI Examination}

1) Obtaining an informed consent by the patient, patient's parents or care givers before MRI examination.

2) Measuring patient body weight for calculation of amount of contrast media.

3) Fasting for 6-8 hours and checking serum creatinine before Gadolinium administration.

\subsubsection{Technique}

Technique had been performed using a 1.5 Tesla unit (GE Signa Explorer).

1) A standard head coil was used.

2) A standard MRI brain was obtained to all the patients. In form of T1- and T2-weighted images and additional sequences as fluid attenuation inversion recovery, diffusion weighted imaging, and diffusion coefficient sequence was obtained.

3) Postcontrast T1W fat saturated sequence was obtained.

4) DTI was then performed.

\subsubsection{Magnetic Resonance Imaging Protocol}

1) The sequences were taken followed by examination started with no delay time and Diffusion Tensor, which composed of a single shot, spin echo echoplanar sequences in 40 encoding direction and a diffusion weighting factors about $800 \mathrm{~s} / \mathrm{mm}^{2}$.

2) DTI measurements were obtained using ROI analysis or tractography .

3) The CST and some of major sub-cortical tracts seen in the phonologic or semantic loop of language - superior longitudinal fascicle and inferior fronto-occipital were reconstructed, defining for each tract separately, the regions of interests (ROIs) around areas of WM that all fibers of each tract has to pass through so that it can reach their cortical or sub cortical stations.

Other parameters:

1. T1WI: TR 450, TE 15, matrix 80 x 81, FOV 230 X177, thickness of slice about $6 \mathrm{~mm}$.

2. T2WI: TR 3612, TE 100, matrix $208 \times 127$, FOV $230 \mathrm{X}$ 177, thickness of slice about $6 \mathrm{~mm}$. 
3. FLAIR: TR 6000, TE 120, matrix 240 x 111, FOV 230 X 184, thickness of slice about $6 \mathrm{~mm}$.

4. DTI: TR 10951, TE 67, matrix 128 x128, FOV 224 X $224 \mathrm{~mm}$, numbers of excitations 2, thickness of slice about: 2.0/00 and flip angle 90 (degrees).

I. All the diffusion-weighted images had been transferred to workstation (Advantage work station 4.7).

II. Images had been processed after that by using the GE soft ware devise for tractography. The maps used were:
a. FA map.
b. ADC map.
c. Directional-encoded color FA maps.
III.3D fibers tractography mapping.

\subsubsection{Diffusion Tensor Imaging Tractography}

1) Images had been analyzed in DTI Studio, to obtain eigenvector and fractional anisotropy (FA) map.

2) For the combination, color mapping had to be generated in association with conventional color coding.

3) Deterministically tractography had been performed on every patient to re-construct sub cortical connection with help of fiber assignment by that continuous track making method.

4) FA thresholds of 0.1 and turning angles 55.8 were defined as criteria to start and stop tracing.

5) The CST and definite major sub cortical tracts had been reformatted, marking for each tract targeted a specific regions of interests (ROIs) in area of WM that most of fibers of each tract has to pass through to reach cortical or sub cortical end stations.

6) DTI tractography images had been reviewed systematically.

7) Tracts were classified after that as displaced , infiltrated, and disrupted. displaced: according to the tract appeared normal or of slight decrease in FA, with abnormally located and/or directed fibers, resulting from remarkable mass displacement.

8) Infiltrated: according to the tract shows reduction in anisotropy yet still identifiable on color maps.

9) Disrupted: according to the tract shows isotropic (or near-isotropic) diffusion, so that tract could not be well identified on directionally color maps.

\subsection{Risks and Ethical Consideration as Definte Provisions to Be Able to Maintain Privacy and Confidentiality of Patients in form of}

1) coding number for each patient with address.

2) Results allowed for research purposes only and not for media announcement.

3) Patient names were hidden while using photos of patients.

4) Techniques and procedures used in study show no conflict with different religions, laws or social rules.

5) written consents had to be obtained from patients included inresearch after explanation of all the benefits and risk of procedure.

\subsection{Statistical Analysis}

Data had been collected, tabled, analyzed statistically by a personal computer with Statistical Package of Social Science (SPSS) by version 20, where following statistics had been applied.

Two types of statistics had been collected:

\subsubsection{Descriptive Statistics e.g. Number (No.), Percentage $(\%)$, Mean $\left(X^{-}\right)$and standard Deviation (SD)}

1) Arithmetic means (x): was used as a measure of central tendency.

2) Standard deviations (SD): was used as a measure of dispersion.

3) Percentages (\%).

4) Median: was used as a measure of central tendency.

5) Range: was used as a measure of dispersion.

\subsubsection{Statistical Analysis}

All data had been entered into Excel sheet Microsoft Office Excel-2007 then analyzed statistically using SPSS Statistical Software (version 20.0.0: IBM Corp: Armonk, NY). Qualitative data was presented by number and percentage. Quantative data presented in form of mean and standard deviation (SD). For categorical variables, Chisquare test was used for analysis. The level of significance was adopted at $p<0.05$. Mean value is sum of all observations divided by number of observation. Standard Deviation (SD) it measures degree of scattered individual varieties around their mean, Sensitivity is the capacity of test to accurately identify affected individuals in the population "true positive". The higher the sensitivity, the lesser the number of unidentified cases "false negatives", Specificity is the capacity of test to accurately exclude individuals who are free of that disease "true negatives". The higher the specificity, the lesser "false positives" will be included and Accuracy (Rate of Agreement $=$ (True positives + True negatives) / Total tested x 100).

\section{Results}

This study included 30 patients; their ages ranged from (560 ) years (mean age is $43.453 \pm 12.238$ years). Among the 30 patients included in our study, of them 24 were males $(80 \%)$ and 6 patients were females $(20 \%)$. (Table 1$)$.

Table 1. Gender distribution of studied cases.

\begin{tabular}{lll}
\hline Gender & N & Studied cases \\
\hline Male & 24 & $80 \%$ \\
Female & 6 & $20 \%$ \\
Total & 30 & $100 \%$ \\
\hline
\end{tabular}

In our study, 8 patients $(26.3 \%)$ had right cystic brain lesion, while 22 patients $(73.3 \%)$ had left cystic brain lesions. (Table 2).

Table 2. Distribution of studied cases according to the side of the lesions. 


\begin{tabular}{ll}
\hline Side of lesion & Studied cases N(\%) \\
\hline Right & $8(26.6)$ \\
Left & $22(73.3)$ \\
Total & $30(100)$ \\
\hline
\end{tabular}

As regard to the Clinical picture there were (12) Patients (40\%) had Headache, 7 patients had Disturbed conscious level, 5 patients had paralysis and 6 patients' complaints of Convulsions. (Table 3).

Table 3. Distribution of studied cases according to clinical picture.

\begin{tabular}{lll}
\hline Clinical picture & N & Studied cases\% \\
\hline Headache & 12 & 40 \\
Disturbed conscious & 7 & 23.3 \\
Paralysis & 5 & 16.6 \\
Convulsions & 6 & 20 \\
\hline
\end{tabular}

As regard to site affected there were 12 Patients (40\%) had Frontal lobe lesions and in 8 patients $(26.6 \%)$ more than one lobe, but affected four patients had temporal affection and 6 patients had parietal lobe affection. (Table 4).

Table 4. Distribution of studied cases according to the site affected.

\begin{tabular}{lll}
\hline Site & N & Studied cases\% \\
\hline Frontal lobe & 12 & 40 \\
Parietal lobe & 6 & 20 \\
Temporal lobe & 4 & 13.3 \\
More than one lobe & 8 & 26.6 \\
\hline
\end{tabular}

As regard 30 patients in this study with 8 malignant lesions
(40\%) and 22 benign lesions (60\%). (Table 5).

Table 5. Distribution of studied cases according to nature of lesions.

\begin{tabular}{lll}
\hline Nature of lesion & N & Studied cases\% \\
\hline Benign & 22 & 60 \\
Malignant & 8 & 40 \\
Total & 30 & 100 \\
\hline
\end{tabular}

There was $20 \%$ of the studied cases had pilocytic astrocytoma, $16.6 \%$ had enlarged perivascular spaces, pyogenic abscess and $13.3 \%$ had porencephalic cyst, cystic brain metastasis and $10 \%$ had cystic glioma and dandy walker. (Table 6).

Table 6. Number and percentage of brain tumors Cases.

\begin{tabular}{lll}
\hline Diagnosis & N & Studied cases\% \\
\hline Enlarged perivascular spaces & 5 & 16.6 \\
Porencephalic cyst & 4 & 13.3 \\
Pyogenic abscess & 5 & 16.6 \\
Cystic glioma & 3 & 10 \\
Pilocytic astrocytoma & 6 & 20 \\
dandy walker & 3 & 10 \\
Cystic brain metastasis & 4 & 13.3 \\
\hline
\end{tabular}

As regard to previous imaging modalities there were 15 patients $(50 \%)$ had CT and 3 patients had MRI $(10 \%)$ and 12 patients $(40 \%)$ did not do previous diagnostic imaging. (Table 7).

Table 7. Distribution of studied cases according to previous imaging.

\begin{tabular}{lll}
\hline Previous imaging modalities & N & Studied cases\% \\
\hline CT & 15 & 50 \\
MRI & 3 & 10 \\
No & 12 & 40 \\
Total & 30 & 100 \\
\hline
\end{tabular}

As regard to previous neurological examination there were 12 patients $(40 \%)$ had motor weakness and 4 patients had language disorder (13.3\%), one case had urinary incontinence, 11 cases had seizure and 2 cases had memory impairment. (Table 7).

Table 7. Distribution of studied cases according to the results of neurological examination.

\begin{tabular}{lll}
\hline Results of neurological examination & N & Studied cases\% \\
\hline Motor weakness & 12 & 40 \\
Language disorder & 4 & 13.3 \\
Urine incontinence & 1 & 3.3 \\
Seizure & 11 & 36.6 \\
Memory impairment & 2 & 6.6 \\
\hline
\end{tabular}

As regard to diffusion tensor imaging there were 14 normal, 8 patients $(26.7 \%)$ had displaced only and 4 patients had invaded (13.3\%) and disrupted was shown in 4 patients $(13.3 \%)$. (Table 8$)$.

Table 8. Distribution of studied cases according to the results of DTI.

\begin{tabular}{lll}
\hline Results of diffusion tensor imaging & N & Studied cases\% \\
\hline Normal & 14 & 46.7 \\
Displaced only & 8 & 26.7 \\
Invaded & 4 & 13.3 \\
Disrupted & 4 & 13.3 \\
Total & 30 & 100 \\
\hline
\end{tabular}

\section{Cases}




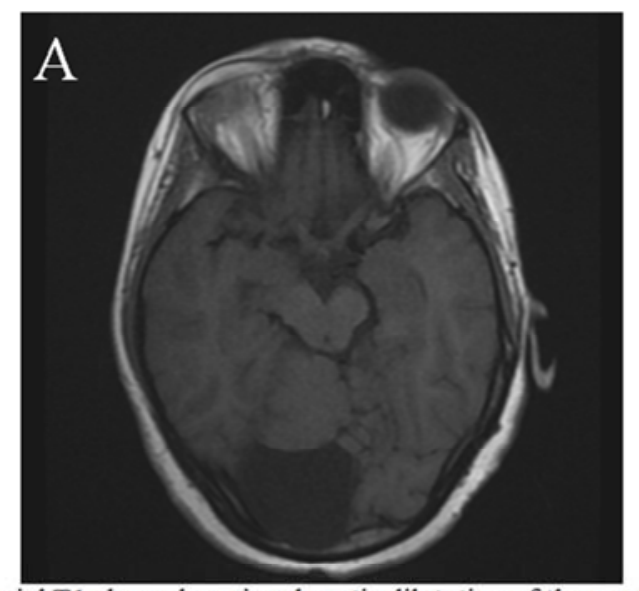

A) Axial T1 shows low signal cystic dilatation of the posterior fossa

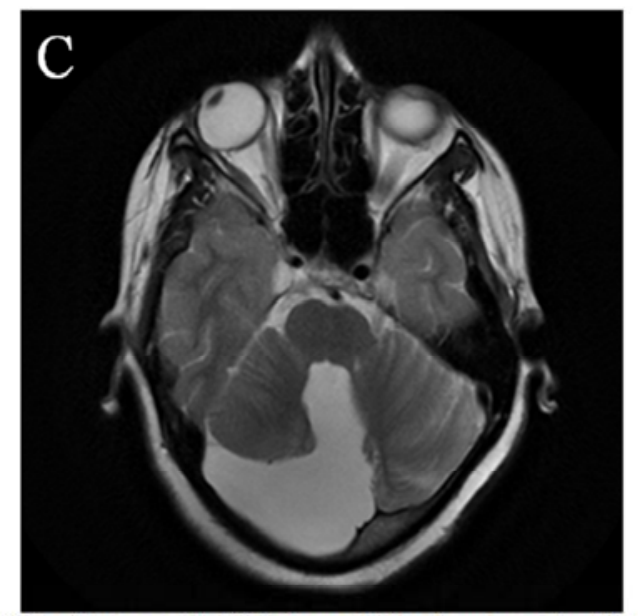

(C\&D) Axial \& sagittal T2 shows high signal cystic dilatation of the posterior fossa extending to fourth ventricle causing its dilatation and right hemi cerebellum is seen hypo plastic

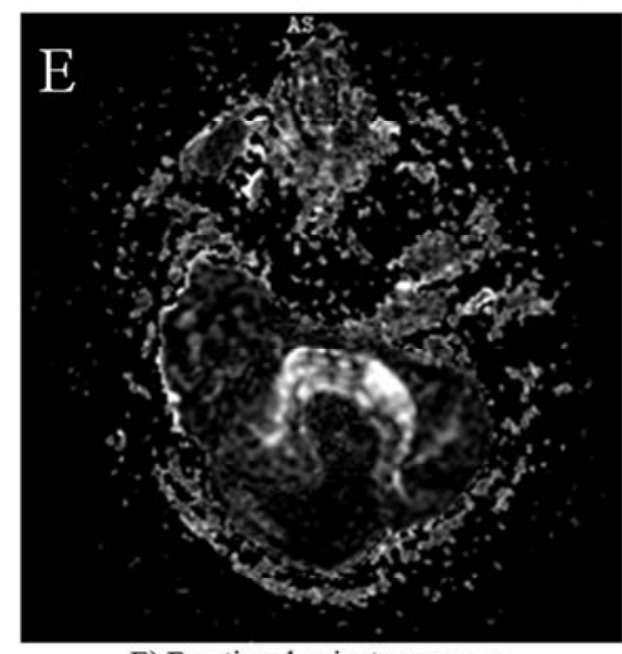

E) Fractional anisotropy map.

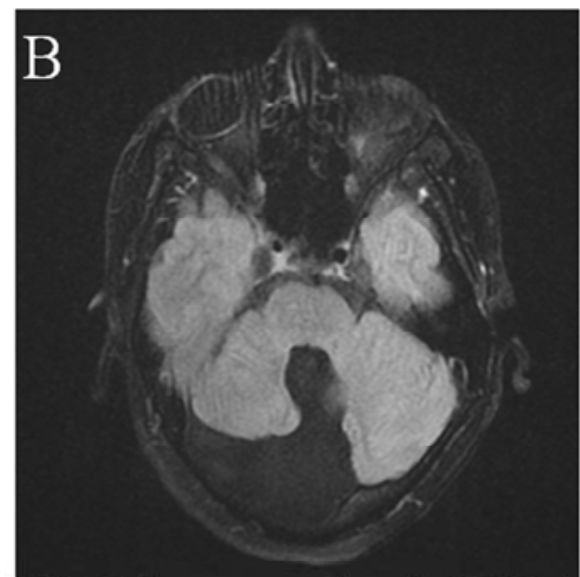

B) Axial FLAIR shows suppression with CSF like signal of cystic area.

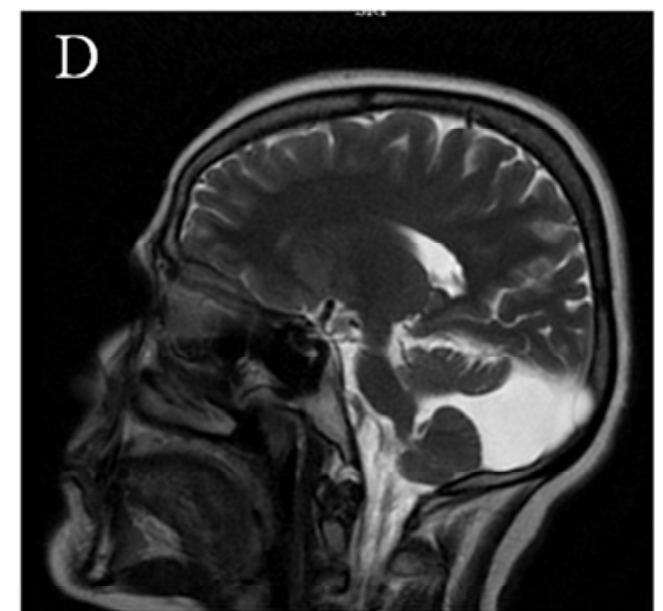

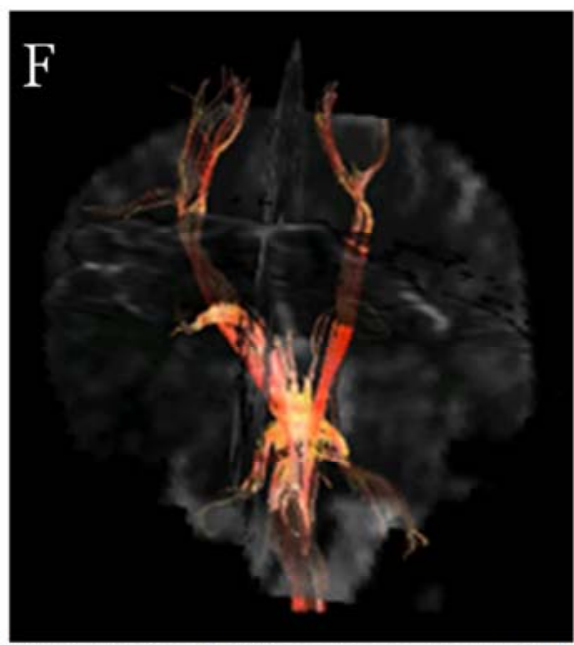

F) 3D tractography showing normal corticospinal tracts.

Figure 1. A case of Dandy walker variant, Female patient 22 years old presented with persistent headache. 


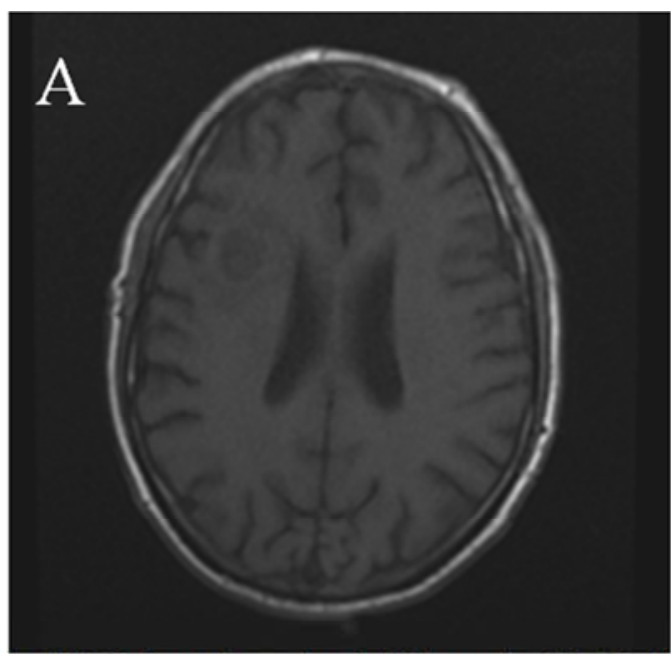

A) Axial T1shows low signal right parietal SOL about $4 \times 3 \mathrm{~cm}$, surrounded by edema with no mass effect detected.

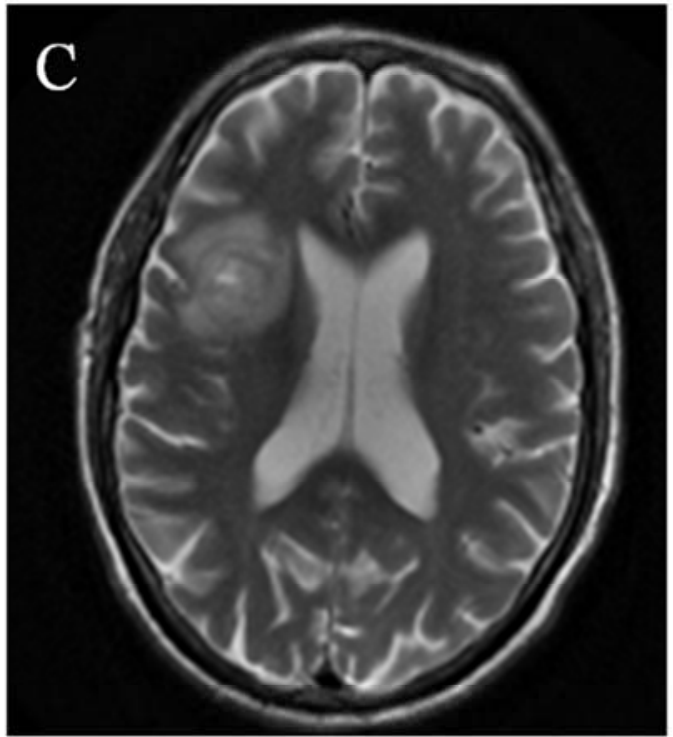

C) Axial T2: high signal with surrounding edema.

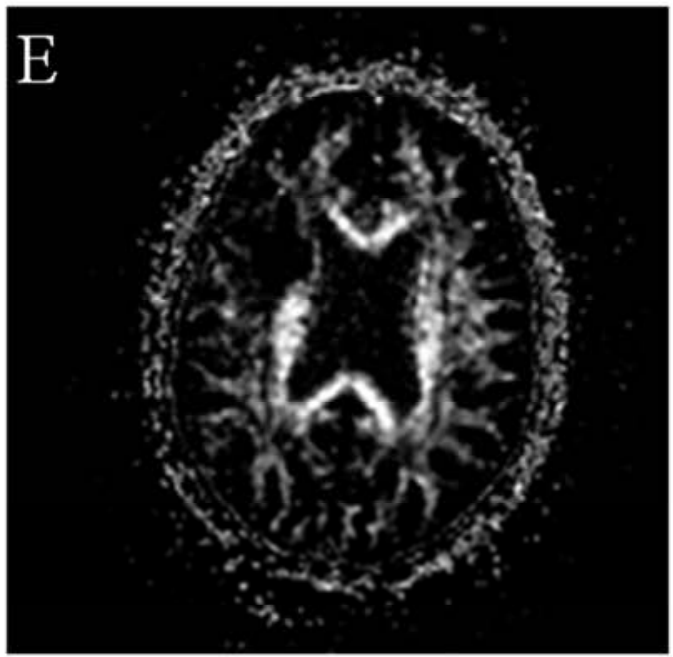

E) Fractional anisotropy map.

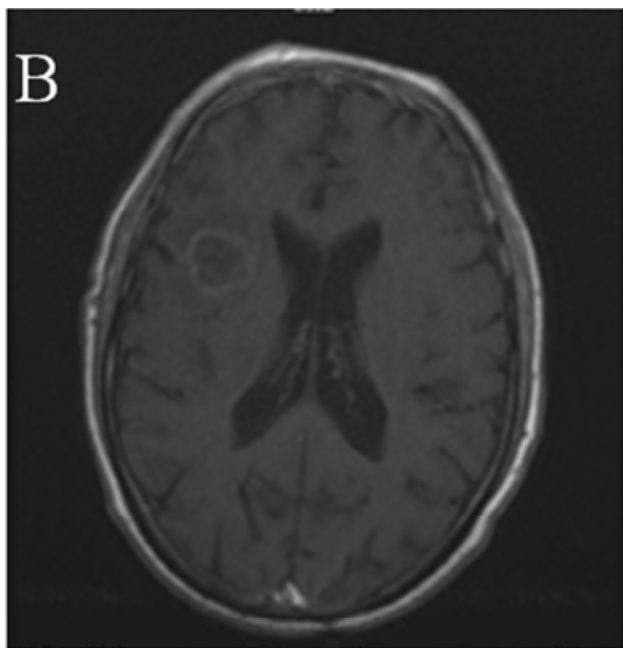

B) Axial T1 with contrast shows ring contrast enhancement of the lesion.

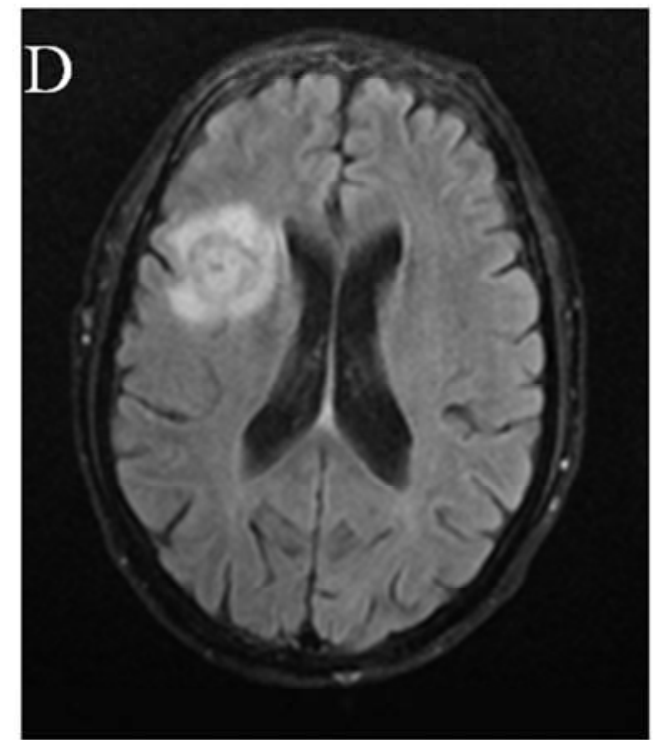

D) Axial FLAIR: high signal with surrounding edema.

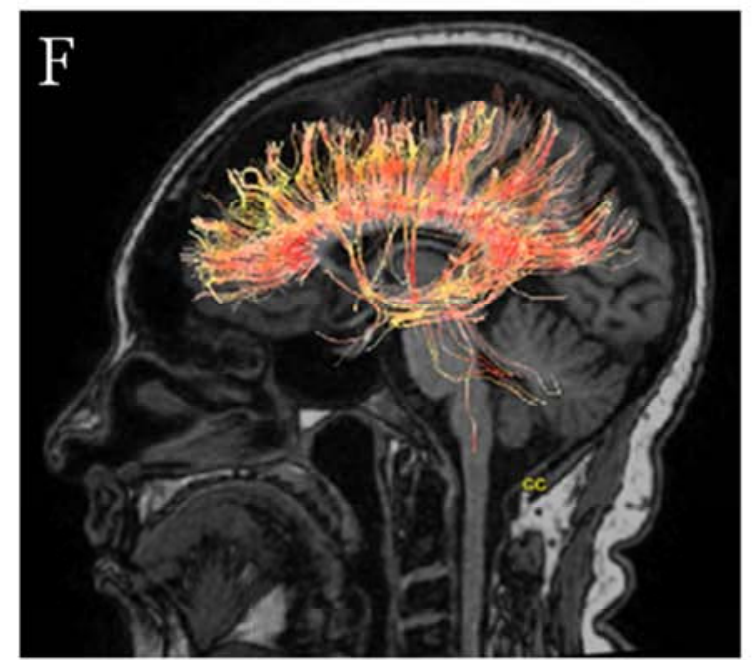

F) 3D tractography showing normal corpus callosum tracts.

Figure 2. A case of right parietal abscess, male patient 57 years old, known chronic renal disease, presented with fever and headache. 


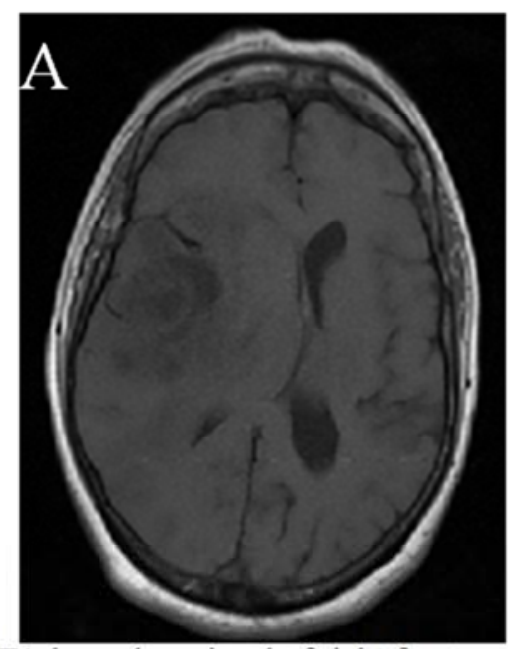

A) Axial T1shows low signal of right frontoparietal SOL with mass effect in the form of effacement of ipsilateral sulci, and compression of ipsilateral lateral ventricle with minimal mid line shift.

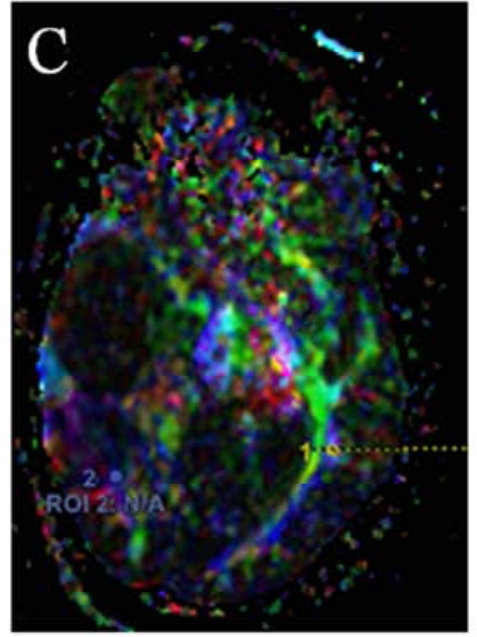

C) axial Color map.

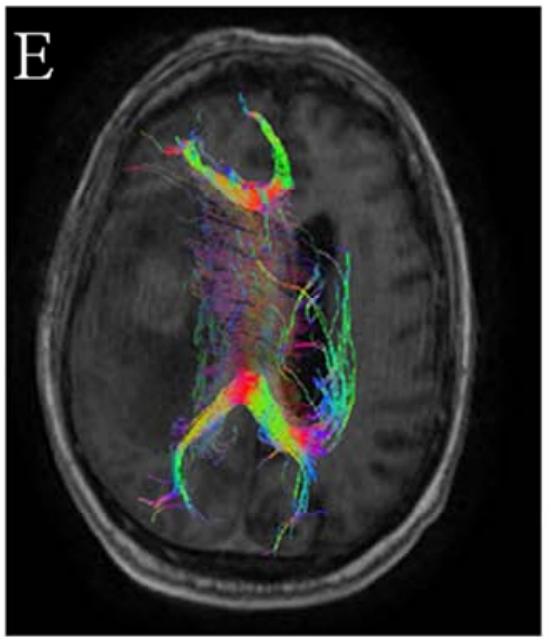

E) 3D tractography shows displaced right cingulum tracts.

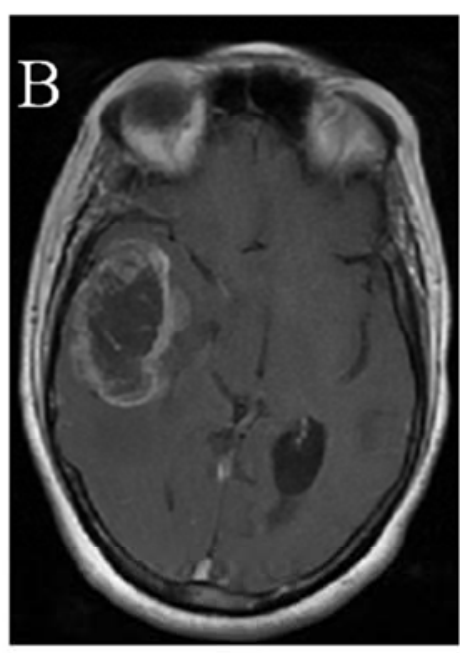

B) Axial T1 with contrast. Showing peripheral non uniform thickness enhancement characteristic of GBM.

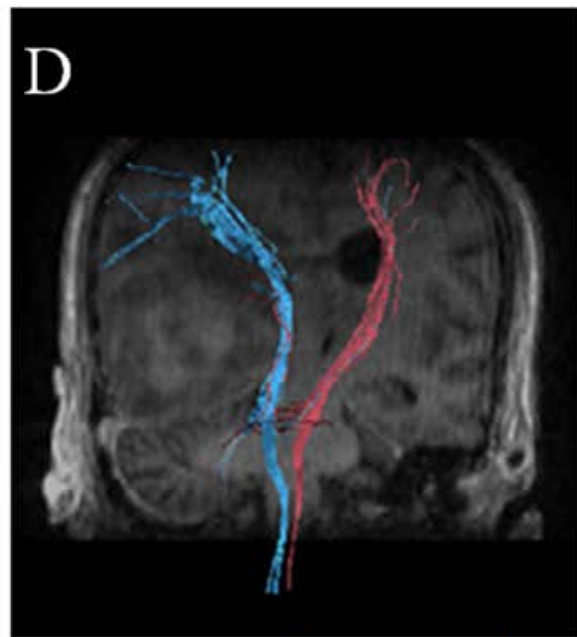

D) 3D tractography on coronal mask shows displaced right corticospinal tracts.

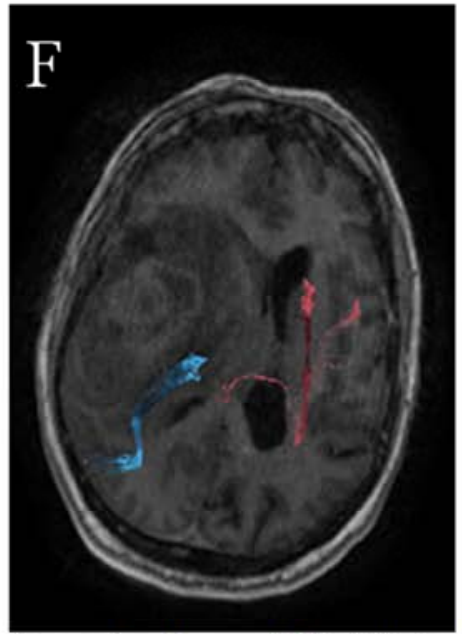

F) 3D tractography shows right infiltrated superior longitudinal fasciculus.

Figure 3. A case of glioblastoma multiform, Male patient 53 years old presented with hemiparalysis of left side following persistent headache. 


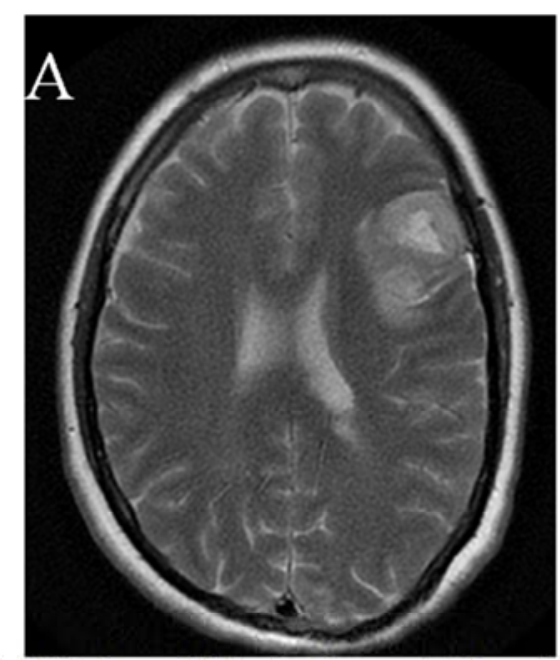

A) Axial T2 shows high signal sounded by edema Left frontoparietal SOL with no mass effect.

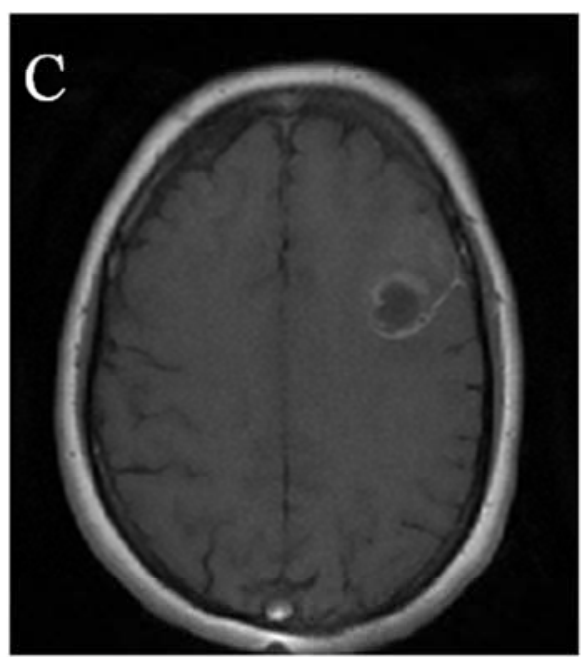

C) Axial T1 with contrast. Showing peripheral non uniform thickness enhancement.

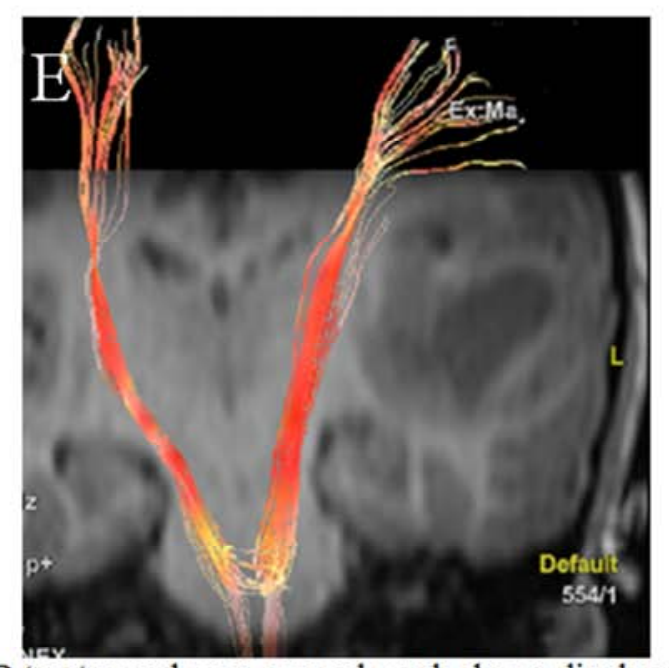

E) 3D tractography on coronal mask shows displaced left corticospinal tracts.

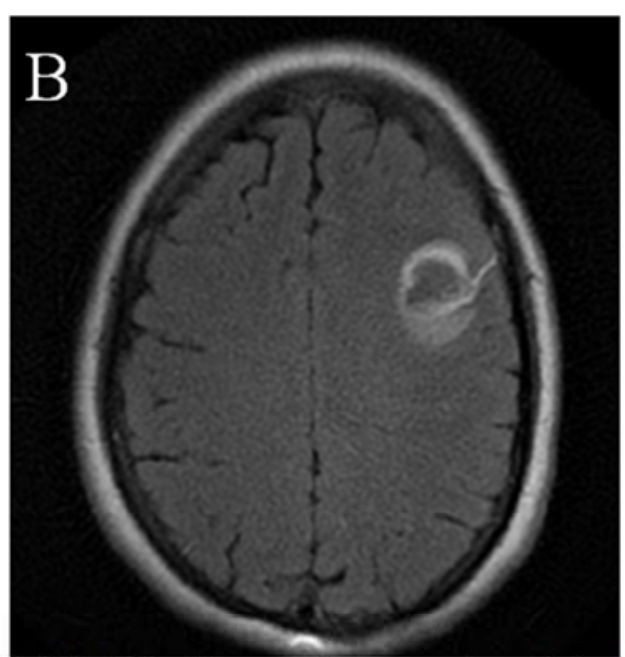

B) Axial FLAIR with central suppression of signal and surrounding edema.

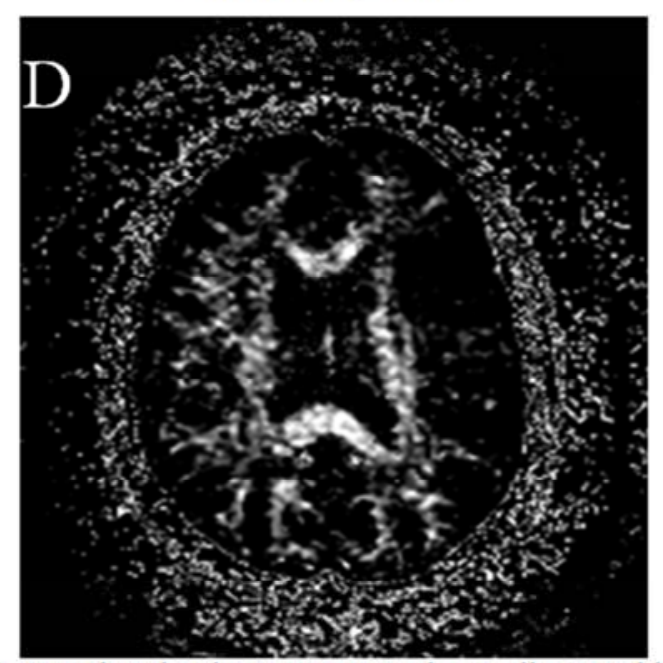

D) Fractional anisotropy map shows disrupted left frontoparietal fibers.

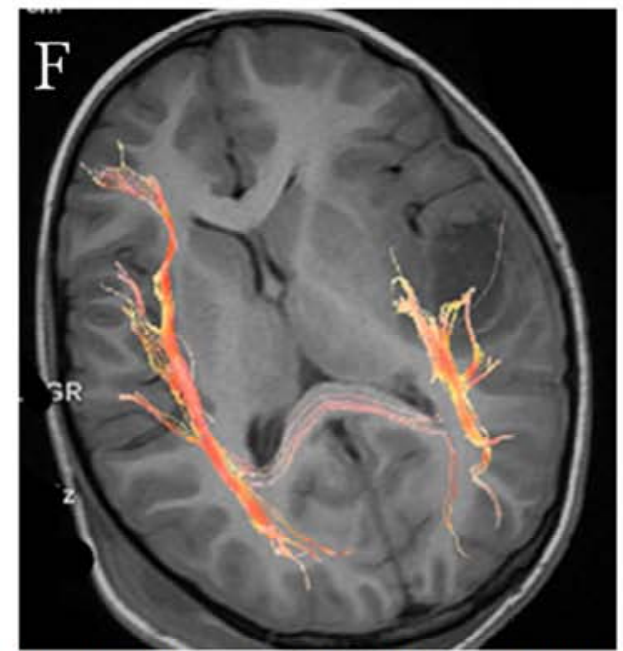

F) 3D tractography on axial mask shows infiltrated left inferior longitudinal fasciculus.

Figure 4. A case of high grade glioma, male patient 36years old presented with seizures. 


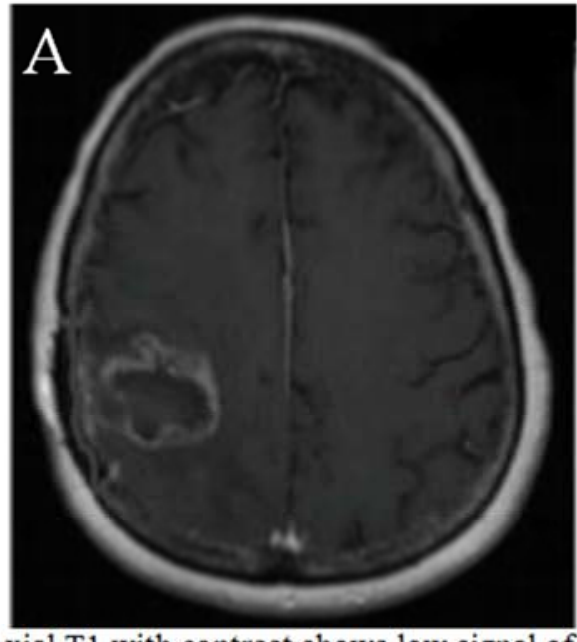

A) Axial T1 with contrast shows low signal of right parietooccipital SOL with mass effect in the form of effacement of cortical sulci.

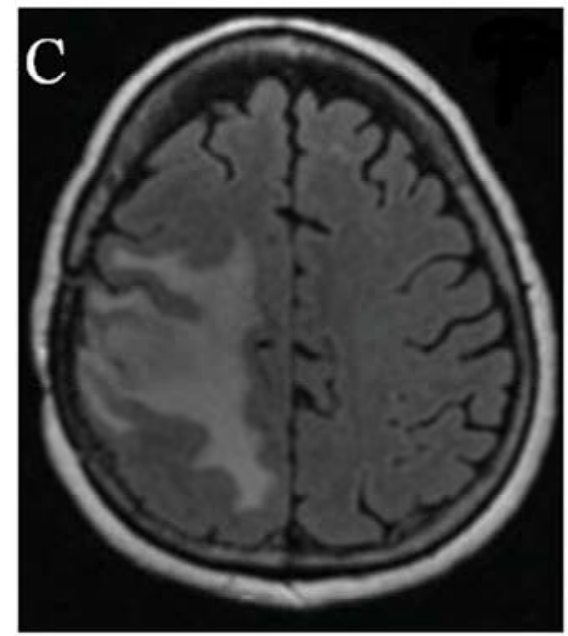

C) Axial FLAIR shows high signal of surrounding edema.

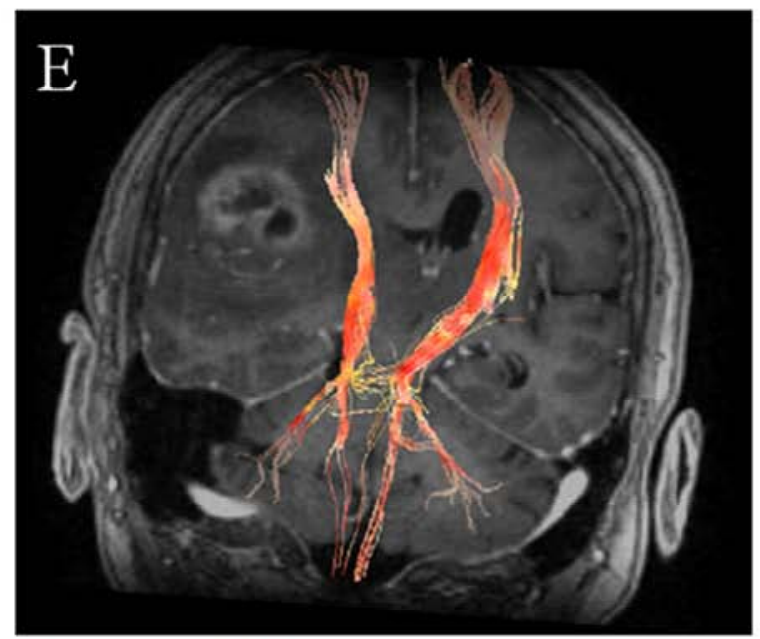

E) 3D tractography displaced right corticospinal tracts.

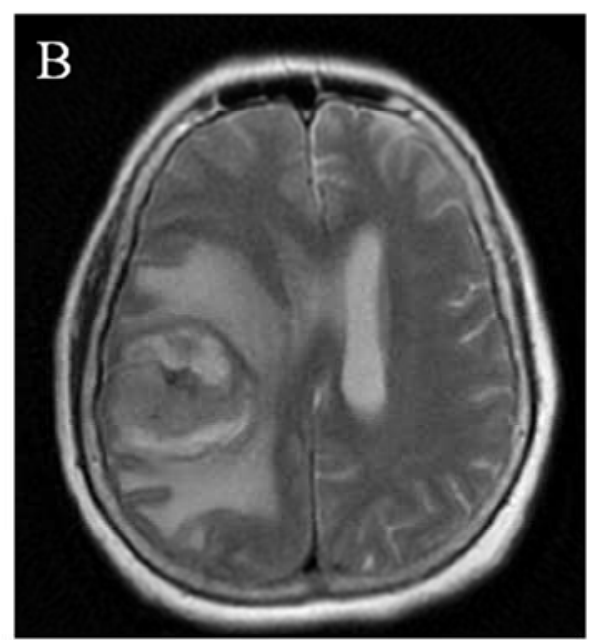

B) Axial T2 shows high signal sounded by vasogenic edema.

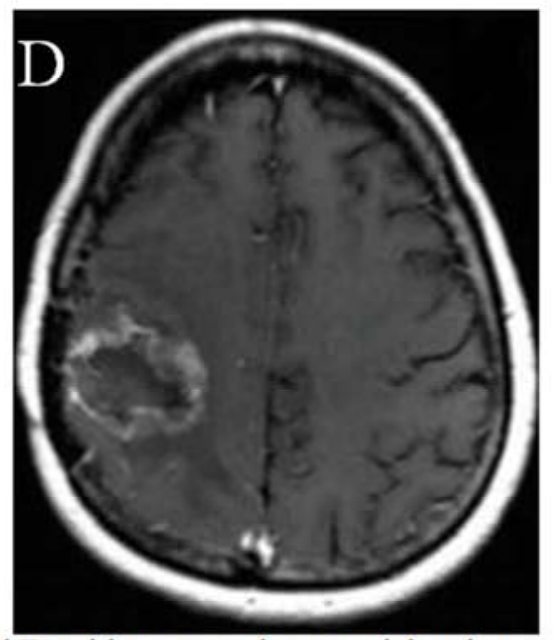

D) Axial T1 with contrast shows peripheral non-uniform thickness enhancement.

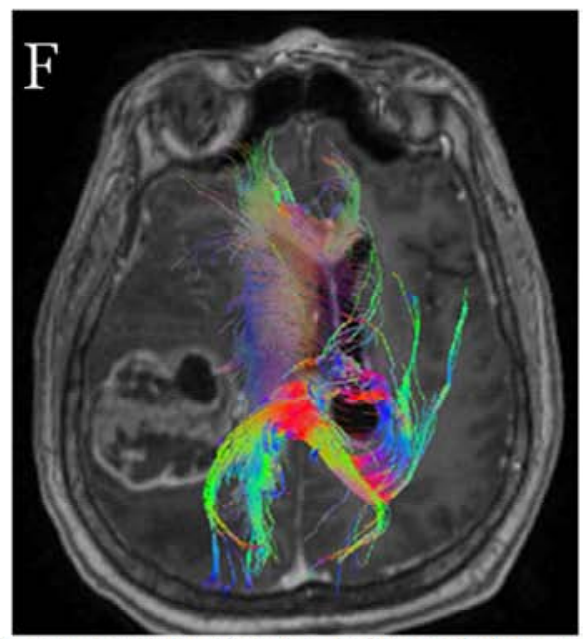

F) 3D tractography showing infiltrated right corpus callosum.

Figure 5. A case of cystic brain metastasis, female patient 42years old, known to have breast cancer, presented with seizures. 

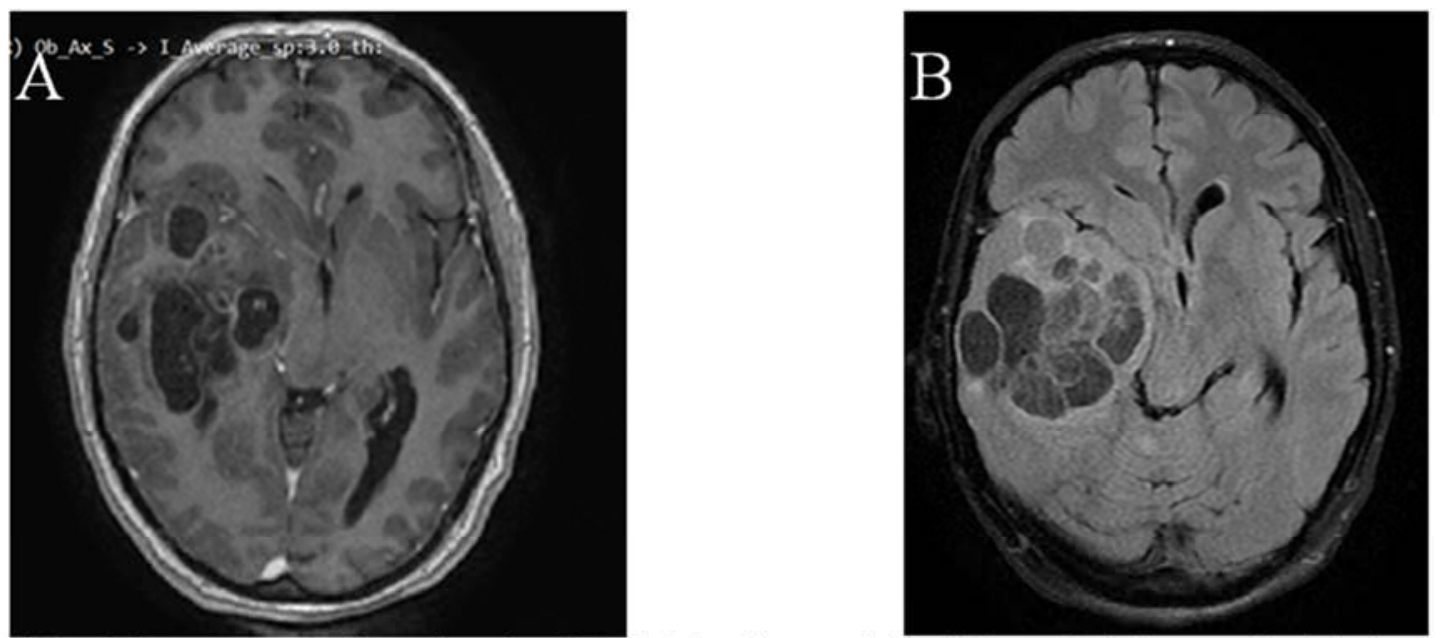

(A)\&(B) Axial T1 \& FLAIR shows low signal Well defined intra axial bubbly cystic lesion seen in right deep parietal region about $6 \times 5 \mathrm{~cm}$, exerting mass effect in the form of effacement of the surrounding sulci, compression of ipsilateral ventricle and mid line shift.

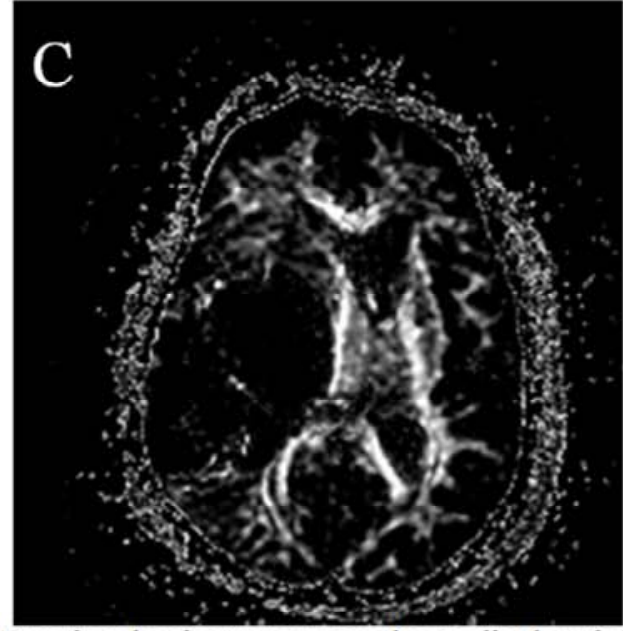

C) Fractional anisotropy map shows displaced right frontoparietal fibers.

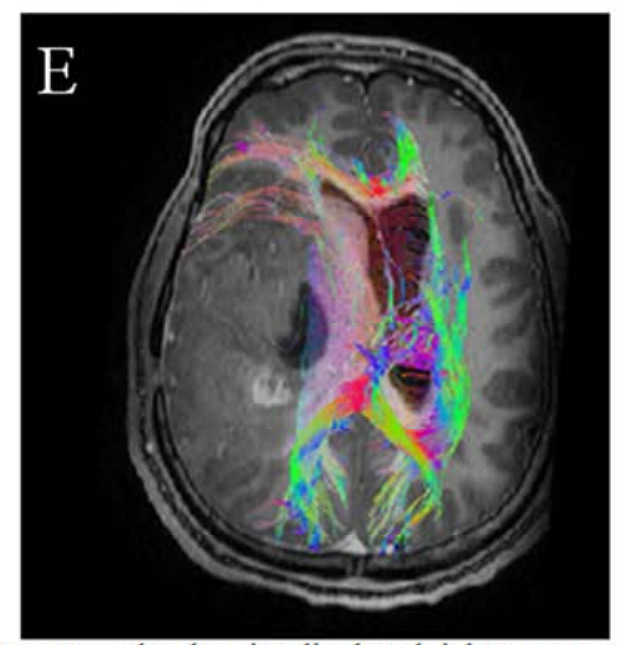

E) 3D tractography showing displaced right corpus callosum tracts.

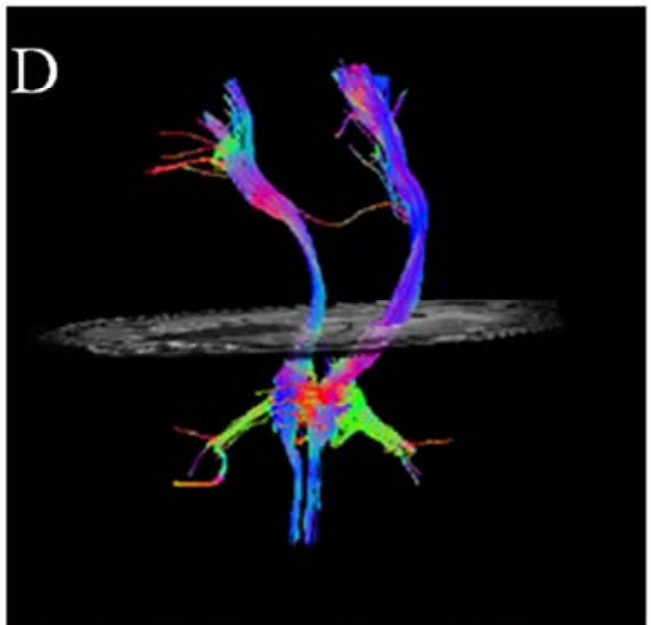

D) 3D tractography showing displaced right corticospinal tracts.

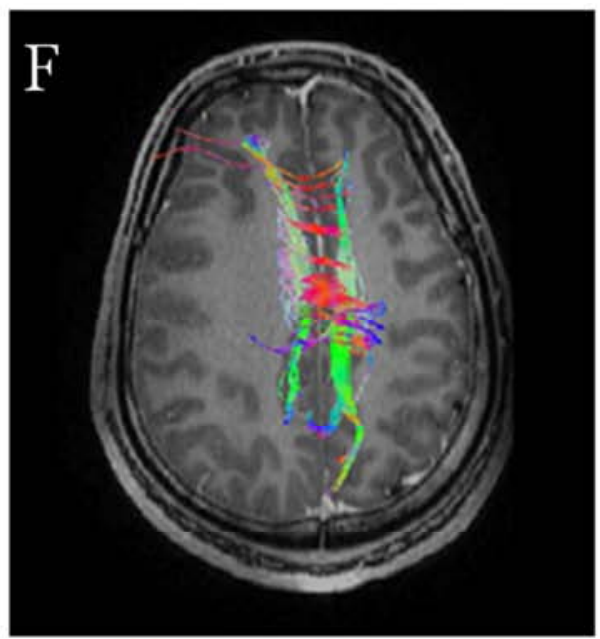

F) 3D tractography showing right displaced cingulum tracts.

Figure 6. A case of cystic tumor recurrence of germinoma, male patient 26 years old, underwent surgical resection for pathologically proved germinoma, presented with vomiting and headache. 


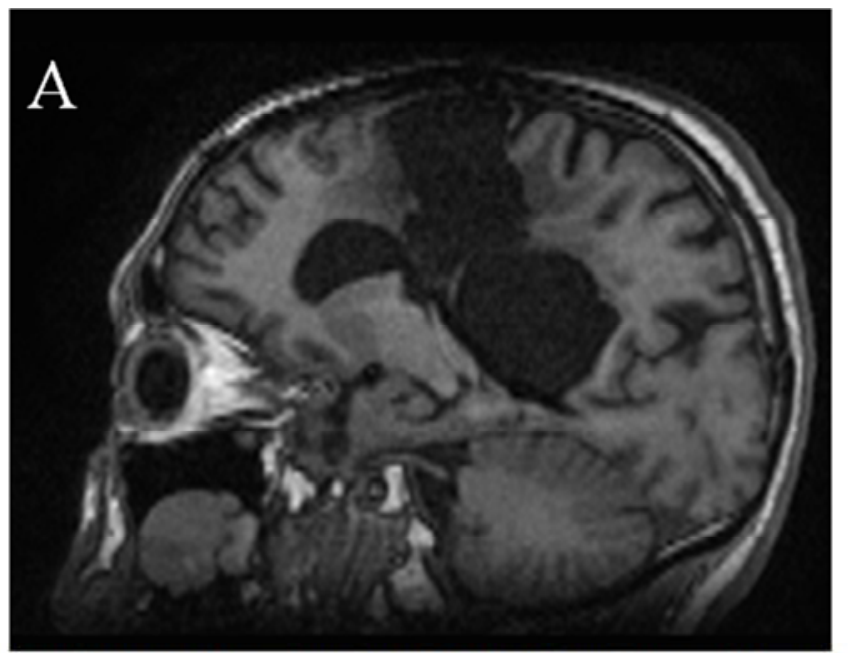

A) sagittal T1 shows low signal Well defined intra axial cystic lesion seen in left parietal region about $5 \times 4 \mathrm{~cm}$, surrounded by gliosis with exvago diltation of ipsilateral ventricle.

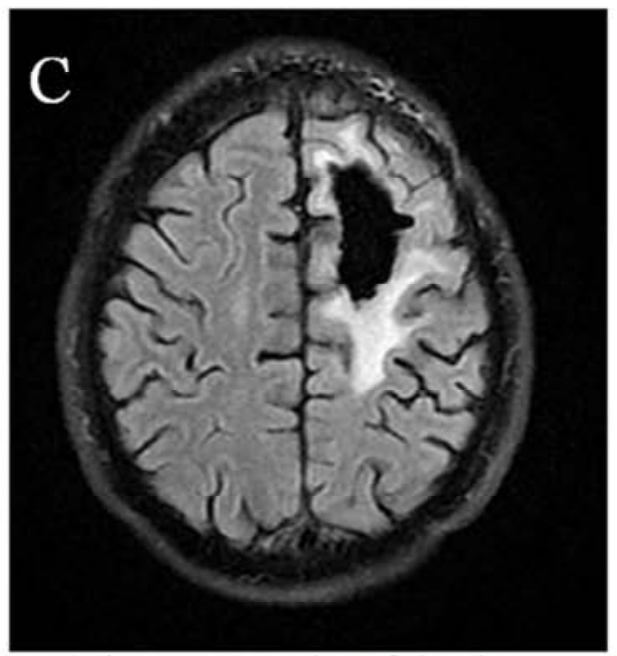

C) Axial FLAIR shows suppression of signal surrounded by rim of high signal of gliosis.

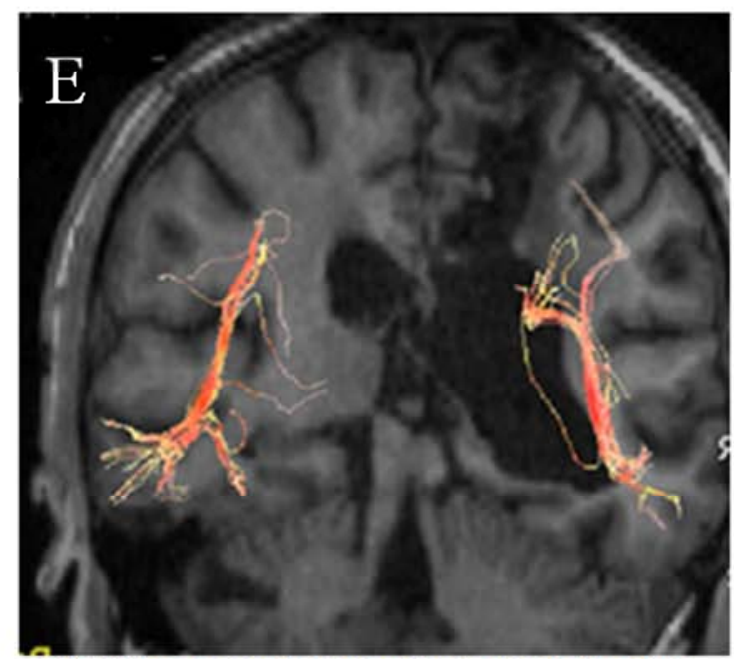

E) 3D tractography showing normal cingulum.

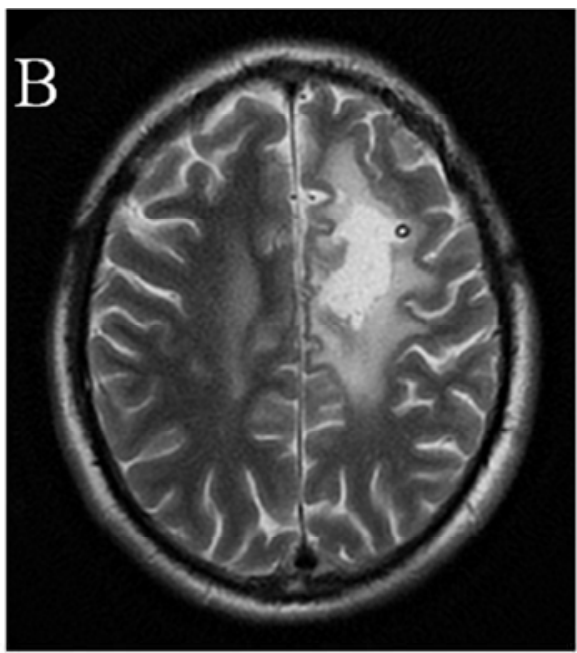

B) Axial T2 shows high signal.

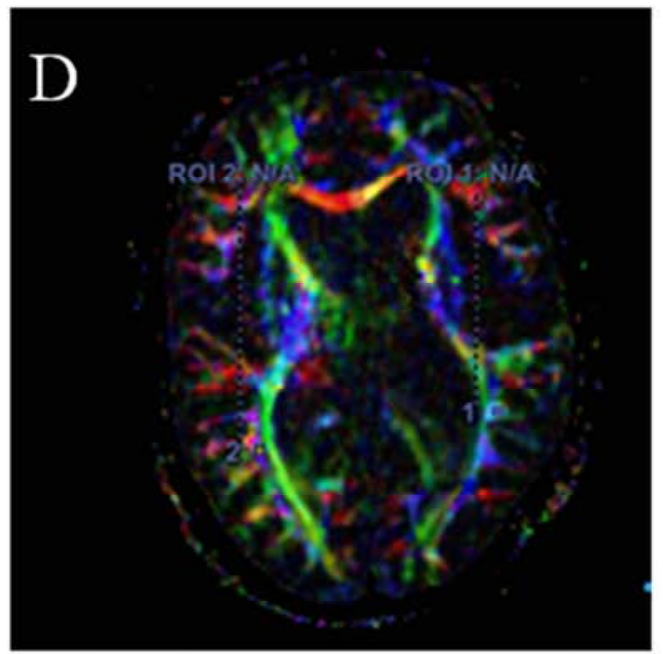

D) Color map shows similar fibers on both sides.

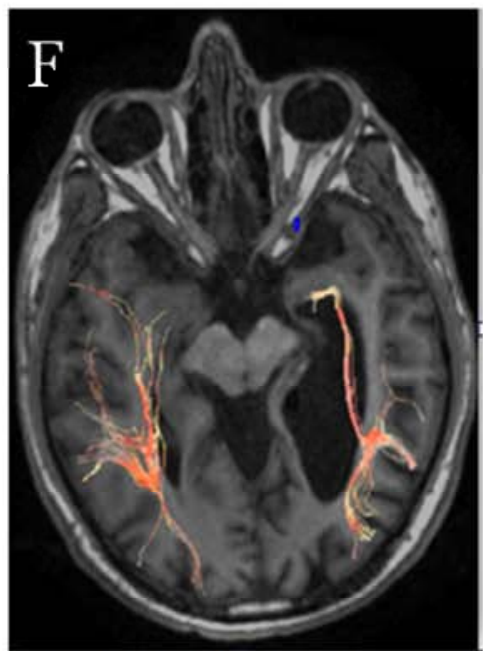

F) 3D tractography showing normal corticospinal tracts

Figure 7. A case of Post-operative cystic encephalomalacia / porencephalic cyst Male patient 46 years old, underwent surgical resection of SOL, presented with seizures. 

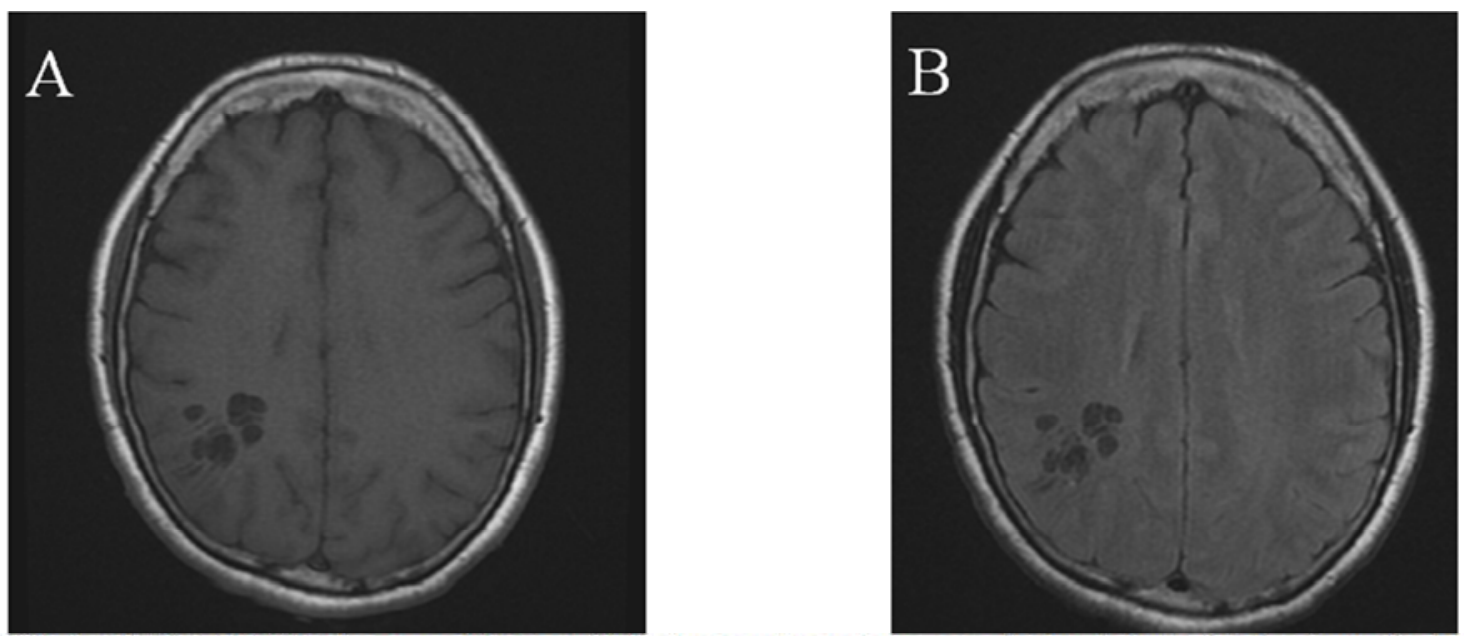

(A)\&(B) Axial T1\& FLAIR shows multiple variable sized cystic lesions, seen in the right parietal region, displaying low signal and CSF suppression.

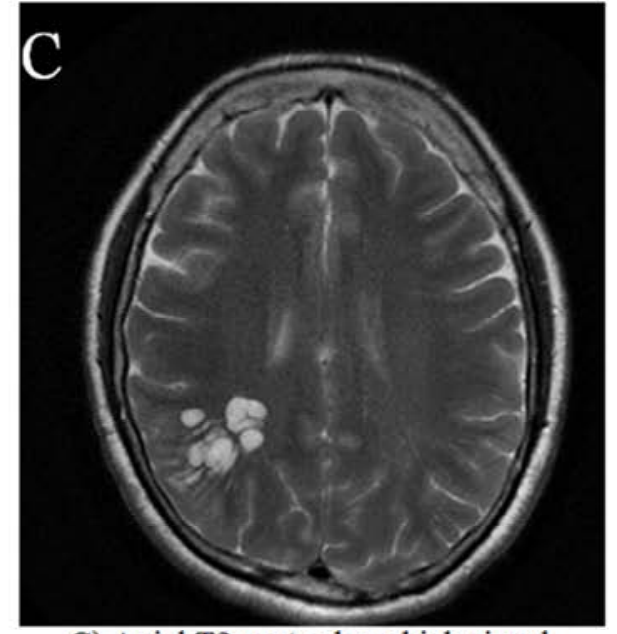

C) Axial T2 cysts show high signal.

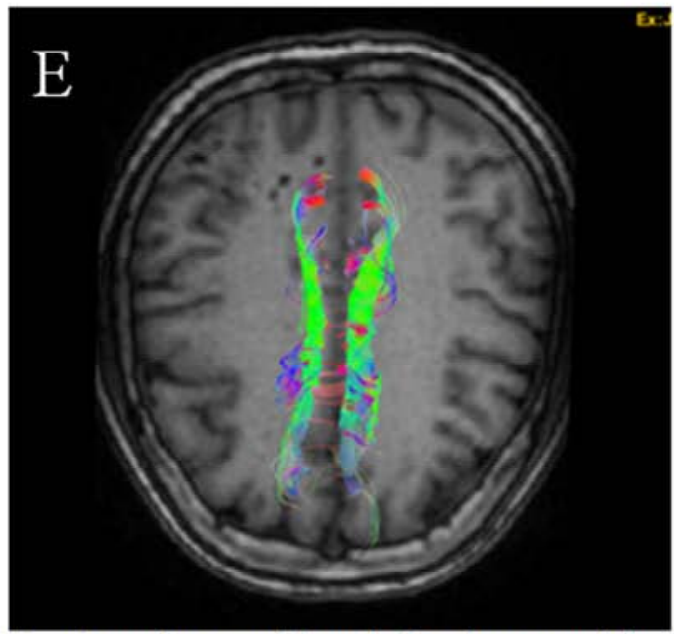

E) 3D tractography on axial mask showing normal cingulum.

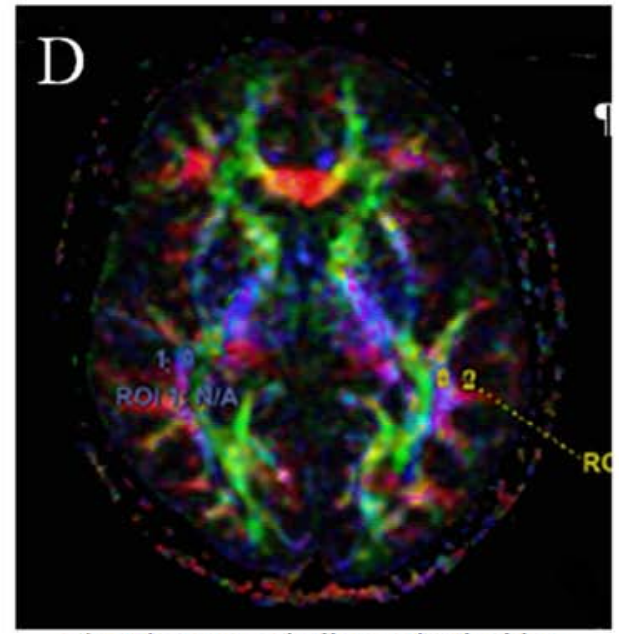

D) Color map: similar on both sides.

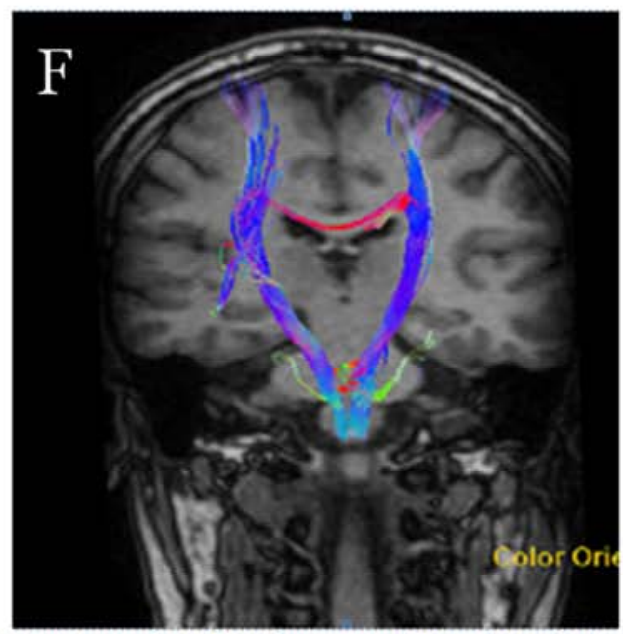

F)3D tractography showing normal corticospinal tracts.

Figure 8. A case of enlarged perivascular spaces (Virchow-Robin spaces), Female patient 40 years old presented with blurring of vision.

\section{Discussion}

Intracranial cystic mass lesions is neuro-surgical problem. Differentiation of multiple cystic brain lesions by $\mathrm{CT}$ and
MRI may be not that easy due to non-specific clinical findings and equivocal radiologic findings [6].

Diffusion tensor imaging (DTI) is a non-invasive magnetic resonance imaging (MRI) facility which characterize the possible location and orientation of sub-cortical white matter 
(WM) tract in vivo [7].

DTI leads surgeons to the relationship between the intraaxial tumor and local WM tracts in different planes. A variable aspects of tumor-tract relationships can, therefore, be explained. Identity of tracts can be explained by its positions and course, such as cortico-spinal tracts(CST) and optic radiations, and proximity of lesion to tracts can be assessed. Position of tumor can be identified the tract, as, superior, lateral, medial, etc., providing definite way to be determined for different areas [8].

Neuro-surgery of brain tumors is comparison between surgical option of resection on one hand and sparing its functions on other hand. Total resection of tumor reduces its risk of recurrence and increase efficiency of radiotherapy or chemotherapy [9].

On other hand, saving functionally relevant zones and so, protecting motor, visual and language functions raise quality of life of those patients [10].

To achieve targeted aims, variable imaging modalities had to be used to evaluate brain tumors, which include MRI, positrons emission tomography, and functional MRI (fMRI) [11].

The aim of work of that current study was to evaluate cystic brain mass lesions by magnetic resonance diffusion tensor tractography. To reach this aim 30 cases subjected to the current study.

In that study mean ages of patients were $43.453+12.23$ years, their age ranged from 5 - 60 years. These were almost similar to study made by Dubey, et al 2018 [12] where ages of participants from 17 to 70 years old with a mean age of 48.3 year old.

As regard to side of cystic brain lesions 8 patients (26.3\%) had right cystic brain lesions while 22 patients $(73.3 \%)$ were with left cystic brain lesion which agrees with Dubey, et al 2018 [13] as $19(55.8 \%)$ patients presented by left sided lesion and $12(35.2 \%)$ patients with right sided lesion.

In the current study showed male predominance $(80 \%)$ which agrees with the study done by Dolecek et al., 2012 [13] as there was male predominance $(68.3 \%)$.

In our study, the most frequent neurological clinical presentation was headache which disagrees with study done by Gomaa and Abdelzaher, 2012 [14] as the most frequent clinical presentation was hemiparesis.

As regard to site affected there were 12 patients $(40 \%)$ had frontal lobes affection and 8 patients $(26.6 \%)$ in more than one lobe, but four of patients (13.3\%) had temporal affection and 6 patients $(20 \%)$ had parietal lobes affection but, in study, done by Chanraud et al., 2010 [7] there were $48 \%$ of patients had frontal lobe affection, $15 \%$ were present in the parietal lobe, and $12 \%$ were present in the temporal lobe. Involvement of more than one lobe was present in $11(25 \%)$ patients.

In current study we classified patients into two groups: Benign and malignant that agrees with Gomaa and Abdelzaher, 2012 [14] who classified patients to two groups: Benign group: with low grade and benign tumors, and, malignant group: with high grade glial tumors, metastasis and lymphoma.

In the current study, we found 8 malignant lesions (40\%) and 22 benign lesions $(60 \%)$ which agrees with Provenzale et al., 2007 [8] who found malignant lesions in $25 \%$ and benign lesions in $75 \%$ of patients.

In this study there were $46.6 \%$ patients had normal $\mathrm{Hb}$ level and $53.3 \%$ patients had low $\mathrm{Hb}$ level which is in agreement with the study done by Romano et al., 2010 [15] who found normal $\mathrm{Hb}$ level in $48 \%$ of their studied patients and low $\mathrm{Hb}$ level in $52 \%$ of their study group.

In current study, there was $20 \%$ of the studied cases had pilocytic astrocytoma, $16.6 \%$ had enlarged perivascular spaces, pyogenic abscess and $13.3 \%$ had porencephalic cyst, cystic brain metastasis $13.3 \%$ and $10 \%$ had cystic glioma and dandy walker which disagrees with Romano et al., 2007 [11] who conducted a study on twenty-five patients. That tumors in study consisted of 11 glioblastomas multiform, six with solitary metastatic lesions, three with stage III glioma, four with stage II gliomas and one with lymphoma. Eight patients had no symptoms related to involvement of white matter tracts studied. In remaining 17 patients, symptoms had been related to affection of pyramidal tract, arcuate fasciculus and optic radiation. They reported that at their follow-up, one patient previously significantly reported speech disorders, likely because of damaging to arcuate fasciculus. In remaining 24 patients, symptoms remained unchanged, with noticed improvement in 14 patients reporting symptoms due to involvement of white matter tracts illustrated in the study [11].

In our study, as regard to previous imaging modalities there were (15) Patients (50\%) had CT and 3 patients had MRI $(10 \%)$ and 12 patients $(40 \%)$ had not done previous imaging modalities which agrees with the study done by Mandonnet et al., 2007 [10] who found that most of the studied cases had previous imaging modalities.

Kleiser et al. 2010 [16] reported that DTI results had been more accurate than when depending on anatomical landmarks only and other imaging modalities, in which fMRI results guided localizing the start points for DTI tract reformatting [16].

Furthermore, in previous study of Romano et al. 2007, they reported that usage of DTI/fMRI in corticectomy resulted in significant change of surgical approach for resection in about $16 \%-21 \%$ of cases [11].

In this study, as regard to Previous neurological examination there were 12 patients $(40 \%)$ had motor weakness and 4 patients had language disorder (13.3\%), a cases had urinary incontinence, 11 cases had seizure and 2 cases had memory impairment but in the study done by Dubey, et al 2018 [12] preoperative neurologic assessment explained that motor weakness noted in $18(52.9 \%)$ patients, language disorders in $3(8.82 \%)$ patients, and $2(5.88 \%)$ patients manifested by urinary incontinence. Twelve patients (35.2\%) showed seizure and $5(14.7 \%)$ patients complained of memory impairment while cognitive disorders reported in $15(44.1 \%)$ patients [12].

The most appreciated use of DTI, in brain tumors is in pre 
operatively confirmation of patency and definite location of displaced WM tracts. WM tracts may be affected by the tumor in many ways. They may be displaced, infiltrated by tumor and/or edema, or destroyed. Unfortunately, these effects are not just confined to a particular tumor or even in a particular WM tract [16].

In comparison between conventional MRI techniques that provide just anatomical information, DTI offers data about central nervous system connectivity by allowing determining crucial WM tracts in brain and identify map for resection operation [17].

In this study as regard to diffusion tensor imaging there were 14 patients $(46.7 \%)$ showing normal DTI, 8 patients $(26.7 \%)$ had displaced only and 4 patients had invaded $(13.3 \%)$ and Disrupted was shown in 4 patients (13.3\%) which agrees with Cruz et al., 2007 [6] in which the authors classified the patterns of white matter tract involvement into displacement, edematous, infiltration and disrupted. Dubey, et al 2018 [12] found that DTI showed the involvement of fiber tracts with a displacement of tracts in almost every patient

Gomaa and Abdelzaher, 2012 [14] in which 32 patients showed intra cranial and extra cranial lesions were reported; they classified patterns of involvement into type I deviation; type II edematous, type III infiltration, type IV destruction. However, it was somehow unlike that classification done by Romano et al., 2007 [11], who divide relationship between tumor and tracts into three types; type I: only displacement, type II: displacement accompanied by disruption, type III: only disruption.

In the current study, there was $46.7 \%$ of the benign cyst had normal diffusion tensor imaging and $13.3 \%$ of the benign cyst had displaced only this agreed with study performed by Romano et al., 2007 [11] In which they clarified displacement pattern in benign and malignant tumors.

Also, in present study, $6.7 \%$ of the benign and malignant cyst had invaded and also $6.7 \%$ of them had disrupted diffusion tensor imaging but in the study done by Romano et al., 2010 [12] destruction reported to be confined to malignant tumors; in both high- and low-grade tumors and atypical meningioma. This signifies that potentially of the DTI to perform efficient role in differentiation between benign and malignant lesions [15].

Pre-operative assessment of damage showed to be possible using fMRI/DTI data in a recent study. A study done by Castellano et al 2012 [18] reported that extent of resection and so, prognosis can be prevoisly expected by DTI. In their study, tumors infiltrated the WM tracts not expected to be resected totally when compared to tumors in which WM tracts proved to be intact [18].

\section{Conclusion}

1) Fundamentally, DTI is the confined method that allows calculation and visualization of fiber tracts in vivo.

2) DTI provides specific information about infiltration of tracts and resulting displaced course by tumor.

3) This study indicates that DTI is an important way in the pre-operative planning of surgery preserving patency of eloquent cortical areas and/or white matter tracts, and so avoid post-operative neurological deficits as involvement of WM tracts is a strong predictor of surgical outcome and provide definition of resection margins.

\section{Abbreviation}

$\mathrm{CT}=$ Computed tomography, $\mathrm{MSCT}=$ Multi slice computed tomography, ADC=Apparent diffusion coefficient, $\mathrm{CST}=$ Corticospinal tract, DTI Diffusion Tensor tractography, DWI=Diffusion weighted image, FA=Fractional anisotropy, FLAIR=Fluid attenuation inversion recovery, MRI=Magnetic resonance imaging, $\mathrm{ROI}=$ Region of interest, WMT White matter tracts.

\section{References}

[1] Lai P., Hsu S., Ding S., et al. Proton magnetic resonance spectroscopy and diffusion-weighted imaging in intracranial cystic mass lesions. Surg Neurol, 2007; 68: 25-36.

[2] Talos IF., Zou KH., Kikinis R., et al. Volumetric assessment of tumor infiltration of adjacent white matter basedon anatomic MRI and diffusion tensor tractography. tumor characteristics. Radiology 2007; 14 (4): 431-621.

[3] Nico S., Alessia F., Haosu Z., et al. Association between clinical outcome and tractography based on navigated intracranial magnetic stimulation in patients with languageeloquent brain lesions. J Neurosurg. 2019; 132 (4): 10331042 .

[4] Oppenheim C., Ducreux D., Rodrigo S., et al. Diffusion tensor imaging and tractography of the brain and spinal cord. J Radiol. 2007; 88 (3 Pt 2): 510-520.

[5] Ilona L, Greg D, Emma C, et al. Tractography in the presence of multiple sclerosis lesions. Neuroimage .2020; 209: 116471.

[6] Lai P., Hsu S., Ding S. et al. Proton magnetic resonance spectroscopy and diffusion-weighted imaging in intracranial cystic mass lesions. Surg Neurol. 2007; 68: 25-36.

[7] Chanraud S., Zahr N., Sullivan EV., et al. A MR diffusion tensor imaging: A window into white matter integrity of the working brain. Neuropsychol Rev, 2010; 20: 25.

[8] Provenzale JM., Mukundan S., Barboriak DP. Diffusionweighted and perfusion MR imaging for brain tumor characterization and assessment of treatment response. Radiology, 2007; 239: 632-649

[9] Smith JS., Chang EF., Lamborn KR., et al. Role of extent of resection in the long-term outcome of low-grade hemispheric gliomas. J Clin Oncol. 2008; 26: 1338-1345.

[10] Mandonnet E., Jbabdi S., Taillandier L., et al. Preoperative estimation of residual volume for WHO grade II glioma resected with intraoperative functional mapping. Neuro Oncol. 2007; 9: 63-69. 
[11] Romano A., Ferrante M., Cipriani V., et al. Role of magnetic resonance tractography in the preoperative planning and intraoperative assessment of patients with intra-axial brain tumours. La radiologia medica. 2007; 88: 906-920.

[12] Dubey A., Kataria R., Sinha VD. Role of diffusion tensor imaging in brain tumor surgery. Asian J Neurosurg. 2018; 13: 302-306.

[13] Dolecek TA., Propp JM., Stroup NE., et al. Statistical report: Primary brain and central nervous system tumors diagnosed in the United States in 2005: 2009. Neuro Oncol. 2012; 14 Suppl 5: v1-49.

[14] Gomaa M. and Abdel Zaher Y. Role of Diffusion Tensor Imaging in Characterization and Preoperative Planning of Brain Neoplasms. Life Sciences. 2012; 9: 163-176.
[15] Romano A., D'Andrea G., Minniti G., et al. Pre surgical planning and MR tractography utility in brain tumour resection. Eur Radiol. 2010; 19: 2798-2808.

[16] Kleiser R., Staempfli P., Valavanis A., et al. Impact of fMRI guided advanced DTI fiber tracking techniques on their clinical applications in patients with brain tumors. Neuroradiology, 2010; 52: 37-46.

[17] Berman J. Diffusion MR tractography as a tool for surgical planning. Magn Reson Imaging Clin N Am 92009; 17: 205214.

[18] Castellano A., Bello L., Michelozzi C., et al. Role of diffusion tensor magnetic resonance tractography in predicting the extent of resection in glioma surgery. Neuro Oncol, 2012; 14: 192-202. 